\title{
ARTICLE
}

\section{A neutron detector for pulsed mixed fields: preliminary measurements}

\author{
Marco Caresana ${ }^{\mathrm{a}}$, Michele Ferrarini ${ }^{\mathrm{b}}$, Giacomo Paolo Manessi ${ }^{\mathrm{c}, \mathrm{d}^{*}}$, Marco Silari ${ }^{\mathrm{d}}$ and Vincenzo Varoli ${ }^{\mathrm{a}}$ \\ ${ }^{a}$ Politecnico of Milan, Department of Energy, Via Ponzio 34/3, 20133 Milan, Italy; ${ }^{b}$ CNAO Foundation, Strada Campeggi 53, \\ 27100 Pavia, Italy; ${ }^{c}$ University of Liverpool, Department of Physics, L69 7ZE Liverpool, UK; ${ }^{d}$ CERN, European Organization for \\ Nuclear Research, 1211 Geneva 23, Switzerland
}

\begin{abstract}
It is well-known that neutron detectors generally suffer from dead-time effects and have strong limitations when measuring in pulsed radiation fields. An active neutron monitor based on $\mathrm{BF}_{3}$ proportional counter and logarithmic electronics was developed to withstand pulsed fields with high intensity, very short duration and high repetition rate. This paper discusses results of preliminary measurements carried out at the Italian National Hadrontherapy Center (CNAO) in Pavia and at the San Raffaele Hospital in Milan. In the first case neutrons were produced by $7 \mathrm{MeV}$ protons accelerated by a LINAC and impinging on a copper Faraday cup. In the second case an electron radiotherapy LINAC produced energy-tunable bremsstrahlung X-rays beams up to $18 \mathrm{MV}$ with an associated neutron component. The neutron monitor was capable to withstand neutron reaction rates up to $2 \cdot 10^{6} \mathrm{~s}^{-1}$ without showing saturation effects. A promising technique is also shown to reject very intense, pulsed gamma fields, due to the innovative operating principle of the detector.
\end{abstract}

Keywords: pulsed fields; $\mathrm{BF}_{3}$; radiotherapy; photoneutrons; LINAC; secondary neutrons, gamma rejection; proportional counter

\section{Introduction}

The need of radiation detectors capable to measure efficiently in pulsed neutron fields is attracting widespread interest for an increasing number of applications such as radiation protection and beam diagnostics [1]. This is a major issue at particle accelerators, where pulsed neutron and gamma fields are present because of beam losses at e.g. targets, collimators and beam dumps. Numerous investigations focused on the development of detectors specifically conceived to work in pulsed fields, but limitations were always present in terms of saturation issues [2], low sensitivity [3] or poor gamma rejection [4].

A promising approach is described by Ferrarini et al. [5], who have discussed the development of a wide dynamic range neutron monitor using $\mathrm{BF}_{3}$ proportional counter. This monitor has a front-end electronics based on a logarithmic amplifier (LogAmp) and, in spite that it works in current mode, it is able to measure a single neutron interaction. This assures a sensitivity comparable to that of commercially available rem counters for steady neutron fields. On the other hand the detector is able to withstand reactions rates in the order of $10^{6} \mathrm{~s}^{-1}$ without showing any saturation effect.

The monitor has been employed for preliminary measurements at the Italian National Hadrontherapy Center (CNAO) in Pavia and at the San Raffaele

*Corresponding author. Email: giacomo.manessi@cern.ch
Hospital in Milan. The aim of these measurements was to test the capability of the detector to discriminate single neutrons, as well as to efficiently measure burst of neutrons in a mixed field with good rejection of the gamma component.

\section{The neutron monitor}

\subsection{Operating principle}

The detector consists of a $\mathrm{BF}_{3}$ proportional counter (length $30 \mathrm{~cm}$, diameter $2.54 \mathrm{~cm}$, gas pressure $700 \mathrm{mmHg}$ (93225 Pa)) placed inside a cylindrical polyethylene moderator with a diameter of $35 \mathrm{~cm}$. The front-end electronics consists of a current to voltage LogAmp, whose output signal is acquired via an ADC and processed with a LabVIEW C program running on a personal computer. The idea of the analysis software is simple: the voltage signal is converted back into a current signal and integrated over a time that can be set by the user. The result of this calculation represents the total charge generated in the $\mathrm{BF}_{3}$ by the neutron interactions. This quantity, divided by the average charge expected by a single neutron interaction, represents the number of neutron interactions occurring during the integration time.

The scheme of the front-end electronics is shown in Figure 1. The polarization between the electrodes of the $\mathrm{BF}_{3}$ counter is provided by an external high voltage 
supply. A fixed current $I_{\min }(200 \mathrm{pA})$ is added to the current signal $I_{R}$ generated by the reactions inside the counter to avoid negative saturation in case of absence of a neutron signal. The sum of the two currents, $I_{R}+I_{\text {min }}$, is collected and processed by the LogAmp. Because the signal is collected at the cathode, the proportional counter must be electrostatically shielded in order to avoid noise pick up. The LogAmp output passes through a cable driver and the signal $\mathrm{V}_{\text {LogOut }}$ is acquired via a digital oscilloscope. For the measurements described in this paper the signal was acquired via a PC oscilloscope (PicoScope ADC 212 by PicoTechnology).

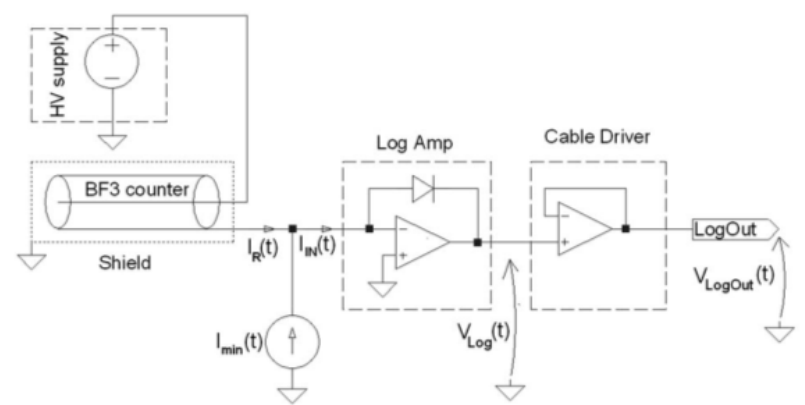

Figure 1. General scheme of the detector electronics.

The choice of the LogAmp was driven by the need to achieve a dynamic range of a few orders of magnitude with respect to the burst intensity to be measured. Conventional LogAmps are too slow, especially in the tail part, for the purpose of the detection of a radiation signal. It was then decided to employ a high-speed LogAmp, the AD8304 (Analog Devices). The small signal bandwidth of older LogAmps is at best a few ten of $\mathrm{kHz}$ while the AD8304 reaches $8 \mathrm{MHz}$.

\subsection{Calibration in charge}

According to the operating principle of the detector, it is of basic importance to perform a calibration in charge, i.e. to calculate the mean collected charge (MCC) expected from a single neutron interaction, expressed in $\mathrm{fC} / \mathrm{n}$, to be used to derive the number of neutron interactions occurring during the integration time.

The calibration was carried out in the CERN calibration laboratory with a PuBe source. The neutron reaction rate was maintained low enough to avoid multiple neutron interactions in the integration window. The average value obtained is $247 \mathrm{fC}$. This value depends on the type of detector and on the HV polarization, which was fixed to $2400 \mathrm{~V}$. Slight differences were noticed when changing the length of the integration window. However, to assure the stability of the MCC value, the length of the window for all the acquisitions was kept equal to the one used during the calibration.

\section{Measurements at CNAO}

\subsection{Acquisition set-up}

A beam of $7 \mathrm{MeV}$ protons was accelerated by a LINAC and impinged on a copper Faraday cup. Each proton pulse had a current of $500 \mu \mathrm{A}$ and a length of $200 \mu \mathrm{s}$. The detector was placed at $4.5 \mathrm{~m}$ from the target in the mixed neutron-gamma field generated by the interaction of the protons in the Faraday cup. The signal was triggered and acquired at a frequency of $12.5 \mathrm{MHz}$.

\subsection{Detector response}

An example of the response signal is shown in Figure 2. It can be noted that the signal lasts for much more than the original $200 \mu$ s of the accelerated proton burst. This is because the neutrons generated in the target are moderated in the polyethylene before interacting in the $\mathrm{BF}_{3}$ counter.

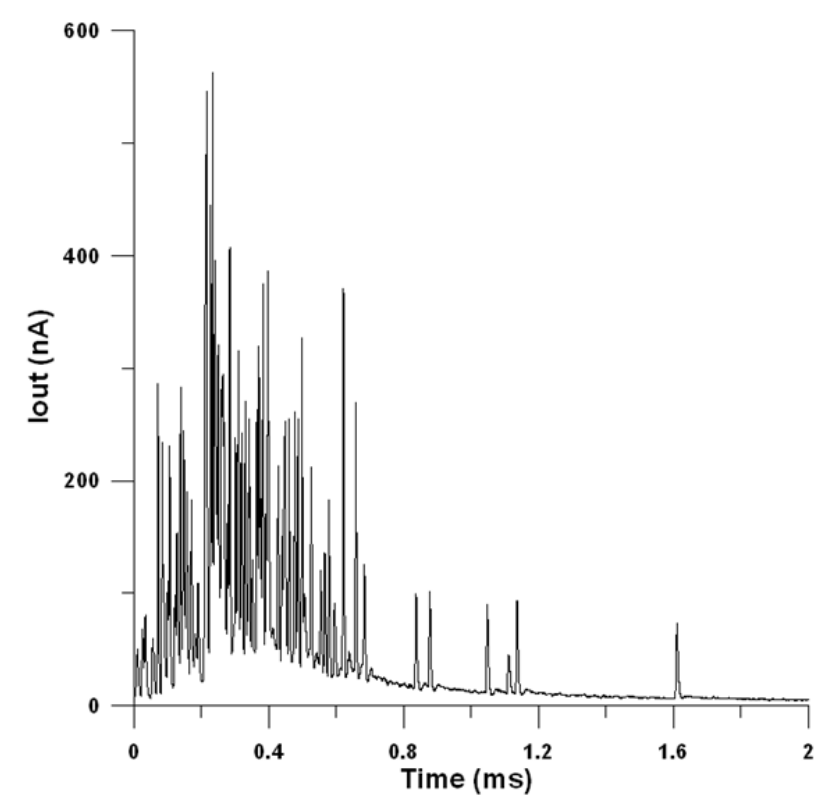

Figure 2. Response signal obtained at CNAO.

The thermalization and drift time of the neutrons in the moderator is in the order of a few hundred $\mu$ s. Most of the interactions occur in fact in the first $500 \mu \mathrm{s}$, and the rare events that occur after $500 \mu \mathrm{s}$ can be ascribed to delayed neutrons.

The total charge generated inside the detector is 93.2 pC. By dividing this value for the MCC calculated before, one obtains 378 neutrons.

Assuming that all the neutrons have been detected in $1 \mathrm{~ms}$, the reaction rate is $3.78 \cdot 10^{5} \mathrm{~s}^{-1}$. This very high reaction rate cannot be detected without losses by a proportional counter coupled to a conventional electronics and data acquisition chain. To estimate the losses that would have occurred with a conventional device, the well-known correction formula for dead-time 
losses for the non-paralyzable model can be applied:

$$
n=\frac{m}{1-m \tau}
$$

where $\mathrm{n}$ is the true interaction rate, $\mathrm{m}$ the recorded count rate and $\tau$ the system dead-time. Assuming a typical dead-time of $5 \mu \mathrm{s}$, and applying the reverse formula, one obtains a recorded count rate of $1.31 \cdot 10^{5} \mathrm{~s}^{-1}, 65 \%$ lower than the true interaction rate. This implies that a proportional counter employed with a conventional acquisition chain would have lost $65 \%$ of the counts.

\section{Measurements at San Raffaele hospital}

\subsection{Acquisition set-up}

An electron radiotherapy Varian Clinac $\mathbb{R}$ DHX Dual energy LINAC. was employed in a fixed irradiation position and the beam directed on the treatment couch, with an irradiation field of $5 \times 5 \mathrm{~cm}^{2}$. The LINAC was operating at an intensity of 600 monitor units (M.U.)/min in two different conditions, producing energy-tunable bremsstrahlung $\mathrm{x}$-rays beams at $6 \mathrm{MV}$ or $18 \mathrm{MV}$. The detector was placed on the therapy couch at $100 \mathrm{~cm}$ from the isocenter. The signal was triggered and acquired at a frequency of $80 \mathrm{MHz}$.

\subsection{Detector response at $6 \mathrm{MV}$}

A response signal of the detector when the LINAC was operating at $6 \mathrm{MV}$ is shown in Figure 3. The signal was triggered by the prompt and intense gamma pulse, which generated a charge in the counter during the first $10 \mu \mathrm{s}$.

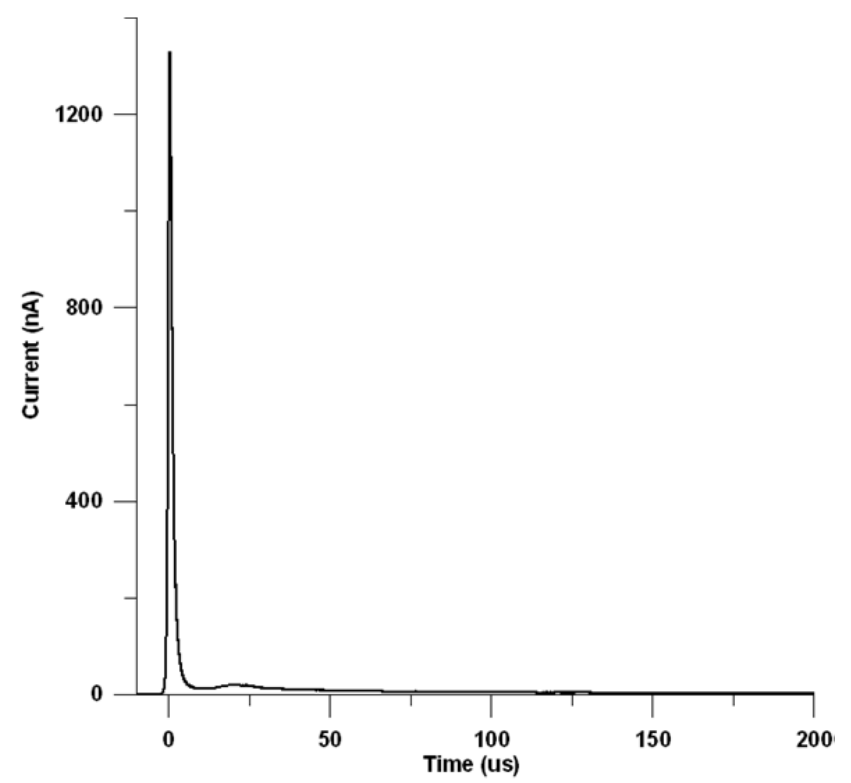

Figure 3. Response signal obtained at the San Raffaele hospital with the LINAC operating at $6 \mathrm{MV}$.
The gamma signal is promptly generated since the photons are not moderated in the detector. The neutron signal is collected later, after the thermalization and drift time in the polyethylene.

The neutron signal is very low and cannot even be reliably quantified, since the current peak generated by the neutrons is two orders of magnitude lower than the gamma current peak. This is not surprising, since the threshold for photoneutron production is for most materials above $6 \mathrm{MeV}$.

\subsection{Detector response at $18 \mathrm{MV}$}

The signal acquired when the LINAC was operating at $18 \mathrm{MV}$ is shown in Figure 4. At this energy the threshold for photoneutron production is well exceeded for most materials employed in the construction of the gantry, e.g. it is $7 \mathrm{MeV}$ for $\mathrm{Ta}$ and $\mathrm{W}$, and $11 \mathrm{MeV}$ for Fe.

The very intense signal detected in the first $10 \mu \mathrm{s}$ after the trigger is due to the prompt gamma-rays. The thermalized neutrons then start to interact in the proportional counter and generate their signal. The signal is more or less constant for the first $300 \mu \mathrm{s}$, and it slowly decreases afterwards.

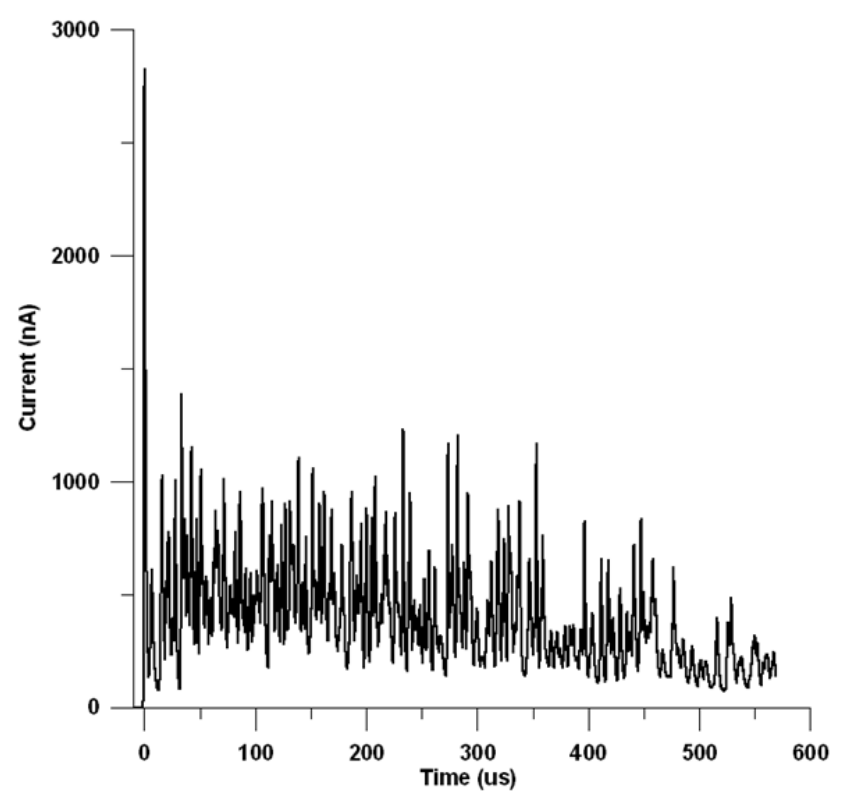

Figure 4. Response signal obtained at the San Raffaele hospital with the LINAC operating at $18 \mathrm{MV}$.

If one calculates the charge generated in the detector by taking into account the whole signal, the gamma contribution would be included in the total value. Due to the fact that the gamma contribution is limited to the first $10 \mu \mathrm{s}$, it can easily be rejected by excluding the first $10 \mu \mathrm{s}$ of the signal from the calculation. This exclusion has no relevance in the calculation of the total number of neutrons that had interacted in the detector, since only a negligible part of them are thermalized in the first $10 \mu \mathrm{s}$. 
By applying this correction, the total charge generated inside the detector is $219.7 \mathrm{pC}$, while the charge generated in the detector in the first $10 \mu \mathrm{s}$ is $6.4 \mathrm{pC}$. By dividing the charge value for the MCC calculated before, one obtains 890 neutrons.

Taking into account that the neutrons were acquired in about $600 \mu \mathrm{s}$, the reaction rate is about $1.5 \cdot 10^{6} \mathrm{~s}^{-1}$. Applying the correction formula (1) for dead-time losses, it can be estimated that a proportional counter with conventional electronics would have lost about $90 \%$ of the counts.

\section{Conclusions}

Preliminary measurements carried out at CNAO (Pavia) and at the San Raffaele Hospital (Milan) have demonstrated that the neutron monitor under study is capable of detecting a neutron reaction rate in mixed fields much higher than what is possible by using a proportional counter whose signal is analyzed by conventional electronics.

Moreover, the detector operating principle allows rejecting efficiently the gamma component unavoidably present in neutron fields, even when this component is very intense and strongly pulsed.

A number of developments are planned to improve the performance of the instrument. Firstly, a reading in terms of ambient dose equivalent would be desirable, to employ the instrument as a rem-counter. This implies the use of a specific moderator that is still being designed. Secondly, the behavior of the detector needs to be analyzed over a wide range of burst intensities in order to evaluate the linearity of its response. Lastly, improvements on the detector sensitivity, on the isotropy of its response and on the signal analysis process can be achieved by employing a ${ }^{3} \mathrm{He}$ proportional counter and by optimizing the electronics.

\section{Acknowledgements}

The authors wish to thank C. Pirovano for the meticulous manual work done in mounting the electronics of the first prototype detector. The authors wish to thank the personnel of the San Raffaele hospital who worked overtime for allowing them to perform the measurements. The authors are also indebted to the CNAO technical staff for providing the necessary support for the measurements.

\section{References}

[1] S. Agosteo, Overview of novel techniques forradiation protection and dosimetry, Rad. Meas. 45 (2010), pp. 1171-1177.

[2] W. Weizhen, L. Jianmin, K. Kejun, A modified neutron dose-equivalent meter for pulsed neutron radiation field, Nucl. Instrum. Meth. A 603 (2009), pp. 236-246.

[3] M. Luszik-Bhadra, E. Hohmann, A new neutron monitor for pulsed fields at high-energy accelerators, Proceedings of the $12^{\text {th }}$ International Congress of IRPA, $19^{\text {th }}-24^{\text {th }}$ October 2008.

[4] K. Iijima, T. Sanami, M. Hagiwara, K. Saito, S. Sasaki, Development of a Current-Readout Type Neutron Monitor for Burst Neutron Fields, Progress in Nuclear Science and Technology 1 (2011), pp. 300-303.

[5] M. Ferrarini, V. Varoli, A. Favalli, M. Caresana and B. Pedersen, A wide dynamic range $\mathrm{BF}_{3}$ neutron monitor with front-end electronics based on a logarithmic amplifier, Nucl. Instrum. Meth. A 613 (2010), pp. 272-276. 